\title{
CONCENTRAÇÕES DE SÓDIO EM BEBIDAS CARBONATADAS NACIONAIS ${ }^{1}$
}

\author{
Cristhiane Caroline FERRARI ${ }^{2}$, Lucia Maria Valente SOARES ${ }^{3, *}$
}

\begin{abstract}
RESUMO
O consumo de bebidas carbonatadas no Brasil é elevado e não existem dados sobre os teores de sódio neste tipo de bebida comercializada no país. O presente trabalho examinou 97 amostras de refrigerantes compreendendo 14 marcas e 7 tipos de bebidas (cola, guaraná, limão, laranja, uva, soda e água tônica). Os teores de sódio encontrados variaram de 19 a $202 \mathrm{mg}$ Na/L. As bebidas adoçadas com açúcar apresentaram uma média de $74 \pm 13 \mathrm{mg} \mathrm{Na} / \mathrm{L}$ e as bebidas com adoçantes artificiais, uma média de $151 \pm 39 \mathrm{mg}$ Na/L. Portanto, os refrigerantes adoçados artificialmente fornecem cerca de duas vezes o teor de sódio das bebidas adoçadas com açúcar. Como conseqüência, um consumidor médio de refrigerantes $(259 \mathrm{~mL} /$ dia) poderá ingerir por dia cerca de $19 \mathrm{mg}$ de sódio provenientes do refrigerante, caso consuma bebidas adoçadas com açúcar enquanto que, o consumidor médio que preferir bebidas do tipo "light" estará ingerindo aproximadamente $39 \mathrm{mg} \mathrm{Na}$ /dia. Já o grande consumidor de refrigerantes (2L/dia) ao preferir os adoçados artificialmente estará ingerindo cerca de $300 \mathrm{mg} \mathrm{Na/dia} \mathrm{provenientes} \mathrm{deste} \mathrm{tipo} \mathrm{de} \mathrm{bebida.} \mathrm{Tal} \mathrm{fato} \mathrm{deve} \mathrm{ser} \mathrm{levado} \mathrm{em} \mathrm{consideração} \mathrm{por} \mathrm{pessoas} \mathrm{com} \mathrm{recomendação} \mathrm{de}$ dieta hipossódica.

Palavras-chave: sódio; bebidas carbonatadas.
\end{abstract}

\section{SUMMARY}

SODIUM CONCENTRATIONS IN BRAZILIAN SOFT DRINKS. High intakes of sodium have been pointed out as one of the causes of high blood pressure. Carbonated drinks are highly consumed in Brazil but there is no data on their levels of sodium. The present work analyzed 97 samples, encompassing 14 brands and 7 types of soft drinks (cola, guaraná, lemon, orange, grape, soda and tonic water). The sodium concentrations varied between 19 and $202 \mathrm{mg} \mathrm{Na} / \mathrm{L}$. The beverages sweetened with sugar exhibited an average of $74 \pm 13 \mathrm{mg}$ $\mathrm{Na} / \mathrm{L}$ while the artificially sweetened drinks had an average $151 \pm 39 \mathrm{mg} \mathrm{Na} / \mathrm{L}$. This means that the "light" carbonated beverages contain twice the amount of sodium of the ones sweetened with sugar. An average soft drink consumer in Brazil (259mL/day) may ingest about $19 \mathrm{mg}$ of sodium from the beverage if he prefers sugar sweetened types and $39 \mathrm{mg} \mathrm{Na} /$ day in case he prefers the light types. On the other hand, a heavy consumer (2L/day) would ingest about $300 \mathrm{mg} \mathrm{Na/day} \mathrm{only} \mathrm{from} \mathrm{soft} \mathrm{drinks} \mathrm{and} \mathrm{this} \mathrm{should} \mathrm{be} \mathrm{taken} \mathrm{into} \mathrm{consideration}$ by persons under low sodium diet.

Keywords: sodium; non-alcoholic beverages.

\section{1 - INTRODUÇÃO}

O sódio é o íon de carga positiva, em maior teor nos liquidos extracelulares do organismo humano. Está em equilíbrio com o íon negativo cloro ou agindo com outros eletrólitos, especialmente o potássio no liquido extracelular, e age como um dos principais fatores de regulação osmótica do sangue, plasma e fluidos intercelulares e do equilíbrio ácido-básico. É essencial para a manutenção do equilíbrio hídrico no interior do organismo, na transmissão dos impulsos nervosos e relaxamento muscular [4].

O sódio é um elemento muito difundido na natureza e o seu teor no corpo humano gira em torno de $1 \%$ do peso do individuo. Ingressa no organismo através dos alimentos e é deliberadamente acrescentado à dieta com o sal de cozinha [4].

A deficiência de sódio pode ser devida a várias causas como ingestão inadequada, perdas excessivas (suor, fluidos pelo trato-gastrintestinal), diuréticos, etc., onde

\footnotetext{
Recebido para publicação em 05/06/2002. Aceito para publicação em 15/05/2003 (000842).

${ }^{2}$ Departamento de Ciência de Alimentos, Faculdade de Engenharia de Alimentos, Universidade Estadual de Campinas.

${ }^{3}$ Departamento de Ciência de Alimentos, Faculdade de Engenharia de Alimentos, Universidade Estadual de Campinas, Caixa Postal 6121, CEP 13081-970 Campinas, S.P. E-mail:valente@fea.unicamp.br

* A quem a correspondência deve ser enviada.
}

podem ser observadas manifestações como fadiga, diarréia, anorexia e hipotensão. Por outro lado, o consumo elevado de sódio na dieta tem sido correlacionado como uma das causas da hipertensão arterial na população. Nos EUA, a hipertensão é a doença crônica mais comum. Trata-se de um problema de saúde que leva a outros mais sérios, como falhas no funcionamento do coração e dos rins [5]. A hipertensão é, no entanto, uma doença de fácil diagnóstico e tratamento eficaz, sem necessidade de recursos técnicos sofisticados, permitindo, portanto, que seu controle possa ser feito na rede básica de serviços de saúde. O controle adequado da hipertensão arterial evita a ocorrência ou mesmo reverte a evolução das complicações decorrentes do nivel elevado da pressão arterial. No Brasil, a hipertensão arterial é uma doença altamente prevalente, atingindo cerca de 15 a 20\% da população adulta com mais de 18 anos, chegando a indices de 50\% nas pessoas idosas [10]. Entretanto, ela não é uma manifestação exclusiva de adultos, pois aproximadamente $1 \%$ a $2 \%$ de crianças e adolescentes podem apresentar hipertensão [7].

Tem sido observado que a tensão arterial média de adultos é baixa, não sobe com a idade e a hipertensão é rara em grupos culturais onde a ingestão de sal é menor que $4500 \mathrm{mg} /$ dia [12]. Outros micro-nutrientes também exercem um papel de controle da pressão arterial: cálcio, potássio, magnésio e cloro [14]. Por outro lado, a manutenção de uma tensão arterial adequada está associada à razão entre sódio e potássio no organismo [11]. 
Apenas parte da população é sensivel ao sódio como causa da hipertensão. Dietas ricas em frutas, verduras e laticinios, mesmo em presença de elevados niveis de sódio, têm sido apontadas como responsáveis pela redução da hipertensão em outros setores da população [8]. Porém, tem-se observado que mais da metade dos pacientes hipertensos são sensiveis a sal na dieta $[5,13]$. Outros fatores como obesidade [9], ingestão de bebidas alcoólicas, envelhecimento, falta de exercício físico, estresse psico-social e tabagismo, contribuem negativamente para o quadro [1]. Além disso, há indícios de que uma dieta rica em cloreto de sódio possa provocar hipertrofia cardiaca $(\mathrm{HC})$, um fator de risco para doenças cardiovasculares [15].

A necessidade mínima estimada de sódio de um ser humano adulto ou em crescimento não excede $500 \mathrm{mg} /$ dia e suspeita-se que possa ser ainda menor [11]. A dieta humana contém habitualmente quantidade adequada para evitar, em condições normais, sintomas de deficiência de sódio. Entretanto é comum adicionar de 6 a 15 gramas de cloreto de sódio diários, como quota suplementar, fazendo com que a dieta possua teores de sódio e cloro acima de suas necessidades, como ocorre em alguns povos em que a hipertensão arterial é encontrada em indice apreciável entre sua população. O processamento de alimentos aumenta o nivel de sódio nos produtos não só pela adição de sal, como é o caso de alimentos salgados, mas também pela adição dos diversos coadjuvantes necessários à modificação de propriedades, tais como, textura, conservação, sabor, etc. Desse modo, um individuo hipertenso deve ser orientado para (1) evitar o consumo de alimentos industrializados, tais como enlatados, conservas, molhos, etc., (2) para não utilizar sal para tempero de alimentos já preparados e (3) ter conhecimento de que alguns adoçantes artificiais contêm sódio e se utilizados com freqüência podem elevar significativamente o consumo do mesmo.

O consumo de bebidas carbonatadas no Brasil é elevado. Em 1998 foram vendidos 20,6 milhões de bebidas não alcoólicas contra 6,3 milhões de litros de sucos de frutas e 16,6 milhões de litros de leite [3]. Isso nos deu em 1998, um consumo per capita de 71,6 litros de bebidas carbonatadas ou assemelhados. Em paralelo, uma preocupação adicional está na crescente popularidade que tais bebidas apresentam entre jovens e familias com crianças pequenas. No Brasil, não existem dados sobre os teores de sódio praticado pela indústria de bebidas. Os niveis de sódio em bebidas carbonatadas no Reino Unido, por exemplo, variam entre 8 e 28mg/100g [6].

O objetivo do presente trabalho foi determinar sódio em bebidas carbonatadas nacionais como uma informação aos profissionais envolvidos com orientação dietética dos diversos grupos populacionais, principalmente aos médicos e nutricionistas que lidam com pessoas com restrições a sódio.

\section{2 - MATERIAL E MÉTODOS}

\section{1 - Amostras}

Foram adquiridas, no comércio local, 2 ou 3 amostras de lotes diferentes de bebidas carbonatadas à base de cola (5 marcas com açúcar e 2 com adoçante artificial), guaraná (7 marcas com açúcar e 3 com adoçante artificial), laranja (4 marcas com açúcar e 1 com adoçante artificial), limão (5 marcas com açúcar), uva (2 marcas com açúcar), soda (3 marcas com açúcar e 2 com adoçante artificial) e água tônica ( 2 marcas com açúcar e uma com adoçante artificial).

\section{2 - Preparo das amostras}

As amostras foram degaseificadas em banho ultrasom e a seguir foram diluidas com adição de solução de lantânio e ácido nítrico (concentrações finais de 0,5\% e $1 \%$, respectivamente).

\section{3 - Preparo de padrões}

Padrões de sódio foram preparados com ampolas de padrão para absorção atômica (Carlo Erba, Merck ou J.T. Baker). As diluições foram feitas com água deionizada (modelo MilliQ plus, Millipore) e as soluções de trabalho continham $1 \%$ ácido nítrico e 0,5\% lantânio.

\section{4 - Amostras de referência}

As seguintes amostras de referência certificadas foram adquiridas do National Institute of Standards and Tecnology, Gaithersburg, EUA: Tomato Leaves, NIST 1573a, Apple Leaves SRM 1515, Non-fat Milk Powder, NIST 1549. As amostras de referência foram digeridas com ácido nítrico, levadas à secura, re-suspendidas em solução de lantânio e ácido nítrico e lidas com corretor de fundo (lâmpada de deutério) na linha de ressonância principal do sódio.

\section{5 - Limpeza de vidraria, recipientes plásticos e demais utensílios}

Após lavagem com água e detergente, foram bem enxaguados e deixados de molho durante a noite em solução $10 \%$ de ácido nítrico, preparada com água destilada. Ao retirar, foram escorridos e lavados pelo menos 3 vezes em água deionizada. Foram então deixados secar em posição invertida e ao abrigo de poeira.

\section{6 - Determinação de sódio}

Foi utilizado o espectrofotômetro de absorção atômica com atomização em chama, marca Perkin-Elmer, modelo 5100PC. As análises foram realizadas usando a linha de ressonância principal do sódio $(589,0$ nm) e pérola de impacto. A altura do queimador foi de $8 \mathrm{~mm}$ e a mistura ar/acetileno foi de 10,0/2,0 (L/ min).

\section{3 - RESULTADOS E DISCUSSÃO}

As amostras de referência certificadas apresentaram diferenças entre o valor certificado e o encontrado abaixo de $10 \%$ para as duas amostras com teores mais elevados de sódio. Com a amostra NIST 1515 a diferença foi de $24 \%$, o que pode ser justificado pelo baixo teor de sódio presente nesta amostra em particular (Tabela 1). 
TABELA 1. Resultados das análises das amostras de referência certificadas.

\begin{tabular}{lccc}
\hline \multicolumn{1}{c}{ Amostra } & $\begin{array}{c}\text { Teor de Sódio } \\
\text { encontrado }(\mathrm{mg} / \mathrm{g})\end{array}$ & $\begin{array}{c}\text { Teor de Sódio } \\
\text { certificado }(\mathrm{mg} / \mathrm{g})\end{array}$ & $\begin{array}{c}\text { Diferença } \\
\text { \%) }\end{array}$ \\
\hline Tomato Leaves, NIST 1573a & $0,1301 \pm 0,0011$ & $0,1360 \pm 0,0040$ & 4 \\
Apple Leaves, NIST 1515 & $0,0321 \pm 0,0014$ & $0,0244 \pm 0,0012$ & 24 \\
$\begin{array}{l}\text { Non-fat Milk Powder, NIST } \\
1549\end{array}$ & $0,497 \pm 0,010$ & $0,5355 \pm 0,0196$ & 8 \\
\hline
\end{tabular}

Resultados indicam a média de triplicatas e o desvio padrão.

As amostras de refrigerantes foram analisadas em duplicatas e cada duplicata foi lida pelo instrumento 3 vezes. Isto possibilitou avaliar a precisão entre preparações distintas de uma mesma amostra e a precisão da leitura do instrumento para uma mesma preparação de amostra. O coeficiente de variação (CV) médio de leituras da mesma preparação da amostra foi 1,6\% e o CV variou entre 0 e 5,4\%. Este dado estabelece a repetibilidade de leituras do instrumento nas condições em que os ensaios foram conduzidos. O CV médio dos resultados das duplicatas das amostras foi 1,7\% e o CV variou entre 0 e 6,2\%. Este último dado indica a repetibilidade do procedimento usado para determinar sódio em refrigerantes no presente trabalho e não se distingue, em termos de ordem de grandeza, da incerteza associada à leitura do instrumento, provavelmente devido à própria simplicidade do procedimento. O limite de detecção para o sódio, calculado como 3 vezes o desvio padrão do branco, baseado em cinco medidas do branco, foi 0,012mg/L.

A necessidade do corretor de fundo para as amostras de refrigerantes foi testada já que estas são amostras com teores baixos de sólidos totais. Os resultados estão na Tabela 2, e mostram que o corretor não é necessário para este tipo de amostras uma vez que as diferenças entre os resultados com e sem corretor estão dentro do erro experimental.
TABELA 2. Comparação do teor médio de sódio entre as amostras analisadas com e sem corretor de fundo.

\begin{tabular}{cccc}
\hline Amostra & $\begin{array}{c}\text { Sem corretor } \\
(\mathrm{mg} / \mathrm{L})\end{array}$ & $\begin{array}{c}\text { Com corretor } \\
(\mathrm{mg} / \mathrm{L})\end{array}$ & $\begin{array}{c}\text { Diferença } \\
(\%)\end{array}$ \\
\hline Refrigerante laranja "light" & 172 & 173 & 0,6 \\
Cola "light" & 56 & 54 & 4 \\
Refrigerante Limão & 149 & 158 & 6 \\
Guaraná & 63 & 60 & 5 \\
\hline
\end{tabular}

Resultados são médias de triplicatas.

Ao todo 14 marcas de refrigerantes foram analisadas. Os teores de sódio encontrados nas amostras de refrigerantes podem ser encontrados nas Tabelas 3 e 4 . As concentrações de sódio encontradas variaram de 19 a 202mg Na/L. As bebidas denominadas "light", por conterem adoçante artificial, apresentam cerca de duas vezes a média de sódio encontrada em bebidas adoçadas com açúcar (Tabela 4). Como conseqüência, um consumidor médio de refrigerantes (259mL/dia) [2] estaria ingerindo por dia cerca de $19 \mathrm{mg}$ de sódio provenientes do refrigerante, caso consuma bebidas adoçadas com açúcar. Por outro lado, o consumidor médio que preferir bebidas do tipo "light" estará ingerindo aproximadamente $39 \mathrm{mg} \mathrm{Na} /$ dia. Já o grande consumidor de bebidas carbonatadas no Brasil (2L/dia) [2] ao preferir bebidas com adoçante artificial estará ingerindo cerca de $300 \mathrm{mg} \mathrm{Na} /$ dia, quando consideramos unicamente refrigerantes como fonte do mineral. Note-se que a ingestão de sódio estimada como minima necessária é $500 \mathrm{mg} /$ dia [11], o que não indica problemas na ingestão de refrigerantes para a população em geral. Já pessoas em dieta hipossódica devem levar em consideração as bebidas carbonatadas como uma fonte de sódio a ser controlada na alimentação.

\section{4 - CONCLUSÕES}

As 97 amostras de refrigerantes nacionais examinadas continham teores de sódio entre 19 e 202mg Na/L.

TABELA 3. Concentrações médias de sódio (mg/L) e desvio padrão em refrigerantes com açúcar

\begin{tabular}{|c|c|c|c|c|c|c|c|c|c|c|c|c|c|c|}
\hline & & & & & Marca & & & & & & & & & \\
\hline Tipo & A & B & C & D & E & $\mathrm{F}$ & G & $\mathrm{H}$ & 1 & $J$ & $\mathrm{~K}$ & L & M & $N$ \\
\hline Guaraná & & $63 \pm 8$ & $45 \pm 2^{\star}$ & $75 \pm 4$ & $106 \pm 2$ & $77 \pm 3$ & $101 \pm 2$ & $70 \pm 1$ & & & & & & \\
\hline Cola & $52+4$ & & & & $73 \pm 3^{*}$ & $20 \pm 1^{*}$ & & & & $72 \pm 3^{*}$ & $42 \pm 2^{*}$ & & & \\
\hline Limão & & & $51 \pm 1^{*}$ & & $96 \pm 0^{*}$ & & $100 \pm 4$ & & $80 \pm 3$ & & & $143 \pm 1^{*}$ & & \\
\hline Laranja & & & & & $98 \pm 8^{*}$ & & $97 \pm 3$ & & & $79 \pm 4^{*}$ & & & & $72 \pm 2^{*}$ \\
\hline Uva & & & & & & & & & & & & & $86 \pm 1$ & $91 \pm 1$ \\
\hline Água tônica & & $64 \pm 2$ & & & & & & & $48 \pm 3$ & & & & & \\
\hline Soda & & $61 \pm 2$ & & & & & & $77 \pm 2$ & $149 \pm 4$ & & & & & \\
\hline
\end{tabular}


TABELA 4. Concentração média de sódio (mg/L) e desvio padrão em refrigerantes com adoçante artificial

\begin{tabular}{|c|c|c|c|c|c|c|c|c|c|c|c|c|c|c|}
\hline \multirow[b]{2}{*}{ Tipo } & \multirow[b]{2}{*}{ A } & \multirow[b]{2}{*}{ B } & \multirow[b]{2}{*}{ C } & \multicolumn{4}{|c|}{ Marca } & \multirow[b]{2}{*}{$\mathrm{H}$} & \multirow[b]{2}{*}{1} & \multirow[b]{2}{*}{$J$} & \multirow[b]{2}{*}{$\mathrm{K}$} & \multirow[b]{2}{*}{ L } & \multirow[b]{2}{*}{ M } & \multirow[b]{2}{*}{$\mathrm{N}$} \\
\hline & & & & D & $E$ & $\mathrm{~F}$ & $\mathrm{G}$ & & & & & & & \\
\hline Guaraná & & $149 \pm 2$ & $151 \pm 1$ & $117 \pm 6$ & & & & & & & & & & \\
\hline Cola & $145 \pm 12$ & & & & & & & & & & $56 \pm 2$ & & & \\
\hline \multicolumn{15}{|l|}{ Limão } \\
\hline Laranja & & & & & & & & & & & & & & $172 \pm 2$ \\
\hline \multicolumn{15}{|l|}{ Uva } \\
\hline Água tônica & & $128 \pm 3$ & & & & & & & & & & & & \\
\hline Soda & & $155 \pm 4$ & & & & & & 202 & & & & & & \\
\hline
\end{tabular}

Os resultados correspondem à média de $2\left(^{*}\right)$ ou 3 amostras de lotes diferentes. Cada amostra, por sua vez, foi analisada em duplicata.

O desvio padrão não indica a incerteza analitica e sim a variabilidade entre amostras.

As bebidas adoçadas com açúcar continham sódio em niveis mais baixos $(74 \pm 13 \mathrm{mg} \mathrm{Na} / \mathrm{L})$ e cerca da metade dos niveis encontrados em bebidas com adoçantes artificiais $(151 \pm 39 \mathrm{mgNa} / \mathrm{L})$. Não há risco de ingestão excessiva de sódio tanto para um consumidor médio como para um grande consumidor deste tipo de bebida. No entanto, pessoas em dieta hipossódica devem ser informadas da presença e teores de sódio em refrigerantes de maneira a possibilitar um melhor controle das fontes do mineral na sua dieta.

\section{5 - REFERÊNCIAS BIBLIOGRÁFICAS}

[1] BEILIN, L.J. Vegetarian and other complex diets, fats, fiber and hypertension. American Journal of Clinical Nutrition, v. 59, p. 1130S-1135S, 1994.

[2] CAMARGO, M.C.R.; TOLEDO, M.C.F.; FARAH, H.G. Caffeine daily intake from dietary sources in Brazil. Food Additives and Contaminants, v. 16, p. 79-87, 1999.

[3] DATAMARK, Trends in the Brazilian packaging industry, http://www.datamark.com.br/frmpg/fstuffe.htm, 09/ 03/2001.

[4] FRANCO, G. Tabela de Composição Química dos Alimentos. 9. ed. Rio de Janeiro: Livraria Atheneu, 1992.

[5] HADDY, F.J.; PAMMANI, M.B. Role of dietary salt in hypertension. Journal of the American College of Nutrition, v. 14, p. 428-438, 1995.

[6] HOLLAND, B.; WELCH, A.A.; UNWIN, I.D.; BUSS, D.H.; PAUL, A.A.; SOUTHGATE, D.A.T. The composition of foods. The Royal Society of Chemistry and Ministry of Agriculture, Fisheries and Food, Cambridge and London, 1991.
[7] KATER, C. E.; COSTA-SANTOS, M. O espectro das sindromes de hipertensão esteróide na infância e adolescência. Arquivos Brasileiros de Endocrinologia e Metabolismo, v. 45, p. 73-86, 2001.

[8] MCCARRON, D.A. Dairy foods in healthy. Bulletin FIL_IDF, v. 336, p. 28-30, 1998.

[9] MCCARRON, D.A.; REUSSER, M.E. Body weight and blood pressure regulation. American Journal of Clinical Nutrition, v. 63, p. 423S-425S, 1996.

[10] MION, Jr. D.; PIERIN, A.M.G.; GUIMARÃES, A. Tratamento da hipertensão arterial - Respostas de médicos brasileiros a um inquérito. Revista da Associação Médica Brasileira, v. 47, p 249-54, 2001.

[11] NATIONAL RESEARCH COUNCIL (NRC), Diet and health: implications for reducing chronic disease risk. National Academy Press, Washington, 1989.

[12] PAGE, L.B. Hypertension and atherosclerosis in primitive and acculturating societies. In: HUNT, J.C. (Ed.) Hypertension update. New Jersey:HLS, 1979. v. 1, p. 1-12.

[13] PREUSS, H.G. Diet, genetics and hypertension. Journal of the American College of Nutrition, v. 16, p. 296305, 1997.

[14] REUSSER, M.E.; MCCARRON, D.A. Micronutrient effects on blood pressure regulation. Nutrition Reviews, v. 52, p. 367-375, 1994.

[15] SANTOS, B.M.R.S.; GONÇALVES, R.M.; PETROIANU, A.; SILVA, A.A.S. Influência de exercícios físicos e do cloreto de sódio na pressão arterial e hipertrofia cardíaca nos ratos. Revista do Hospital das Clínicas da Faculdade de Medicina de São Paulo, v. 54, p. 111-114, 1999. 